\title{
Laparoscopic uncut Roux-en-Y for radical distal gastrectomy: the study protocol for a multirandomized controlled trial
}

This article was published in the following Dove Press journal:

Cancer Management and Research

\author{
Quan Wang' \\ Qingrong $\mathrm{Ni}^{1}$ \\ Kelu Yang' \\ Sheqing $\mathrm{Ji}^{2}$ \\ Yong Fan $^{3}$ \\ Chen Wang ${ }^{3}$ \\ Wenbin Zhang ${ }^{4}$ \\ Su Yan ${ }^{5}$ \\ Qi $\mathrm{Ma}^{2}$ \\ Qiuya $\mathrm{Wei}^{3}$ \\ Di Zhang' \\ Juan $\mathrm{Yu}^{\prime}$ \\ Gang Ji'
}

'Department of Digestive Surgery, Xijing Hospital of Digestive Disease, Xijing Hospital, The Fourth Military

Medical University, Xi'an 7I0032,

China; ${ }^{2}$ Department of General

Surgery, Henan Cancer Hospital

\& Zhengzhou University Cancer

Hospital, Zhengzhou University,

Zhengzhou 450003, China;

${ }^{3}$ Department of Minimally Invasive

Surgery, The Second Hospital

of Lanzhou University, Lanzhou

University, Lanzhou 730000, China;

${ }^{4}$ Department of Gastrointestinal

Surgery, The First Affiliated Hospital

of Xinjiang Medical University,

Xinjiang Medical University, Urumqi

830000 , China; ${ }^{5}$ Department of

Gastrointestinal Surgery, Affiliated

Hospital of Qinghai University, Qinghai

University, Xining 810000, China

Correspondence: Gang Ji

Xijing Hospital of Digestive Disease, Xijing

Hospital, The Fourth Military Medical

University, 127 Changle West Road,

Xincheng District, Xi'an 710032, China

Email jigang@fmmu.edu.cn

\begin{abstract}
Gastric cancer is the third most common cause of cancer-related deaths and is the fifth highest incidence of cancer worldwide, especially in Eastern Asia, Central and Eastern Europe, and South America. Currently, surgery is the only curative treatment for gastric cancer; however, digestive tract reconstruction after distal gastrectomy for gastric cancer is controversial due to the postoperative complications such as reflux gastritis. There is an increasing trend toward laparoscopic uncut Roux-en-Y (URY) for radical gastrectomy. However, evidence on the feasibility of this procedure in patients undergoing laparoscopic radical distal gastrectomy is still absent. Thus, a prospective randomized trial is warranted. This is a prospective, multicenter, two-arm randomized controlled trial in which 210 patients will be randomly assigned to two groups: laparoscopic URY ( $\mathrm{n}=105)$ and laparoscopic Billroth II plus Braun anastomosis $(\mathrm{n}=105)$. Each participant must be pathologically diagnosed with gastric cancer and undergo laparoscopic radical gastrectomy at Xijing Hospital and other four hospitals. The laparoscopic URY procedure is based on the Billroth II gastrojejunostomy plus Braun anastomosis, and then blocked the jejunum input loop at the stump-jejunal anastomosis. The patients' demographic and pathological characteristics will be recorded. The total and oral nutritional intake, general data, total serum protein, serum albumin, blood glucose, and temperature will be recorded before surgery and at the time of hospitalization. Postoperative adverse events will also be recorded, as well as at follow-up appointments at three months and six months after surgery. The rate of reflux gastritis will represent the primary endpoint, and other secondary endpoints, which are all recorded.
\end{abstract}

Keywords: Billroth II gastrojejunostomy, Braun anastomosis, jejunum input loop, anastomosis, reflux gastritis

\section{Introduction}

Gastric cancer is one of the most common malignant cancers, which is fifth incidence worldwide and third most common in terms of tumor-related death. ${ }^{1}$ The morbidity of gastric cancer is higher in Southeast Asian than in European and American regions. This includes China, which accounts for $\sim 40 \%$ of patients with gastric cancer around the world, and this number is increasing. It has been estimated that $>670,000$ new cases of gastric cancer were diagnosed in China in 2015. The majority of times, the lesion is located in the lower stomach, and the distal gastric carcinoma is the major pattern in the Southeastern Asian region such as China, Japan, and South Korea. The therapy is surgery based and is an effective treatment strategy. At present, the proposal is generally changed from subtotal gastrectomy to systematic lymph node dissection. The best treatment option for lower gastric cancer is considered to be 
radical distal gastrectomy. However, none of the traditional surgeries, Billroth I, Billroth II, modified Braun anastomosis, or Roux-en-Y anastomosis, could eliminate the postoperative complications, such as bile reflux. And an optimal technique has not yet been established for surgeons. ${ }^{2}$ With the development and evolution of surgery technology, the effectiveness of gastrectomy for patients with gastric cancer has been remarkably improved, especially the quality of life and survival time. However, the optimum choice of a digestive tract reconstruction procedure after gastrectomy is still controversial. Generally speaking, for surgeons the Billroth I anastomosis is technically simple and widely applied, but its limitations are that anastomosis produces heavy tension and has a relatively high recurrence risk. ${ }^{3}$ While Billroth II could avoid the issue of the heavy tension, this procedure transforms the normal digestive pathway and therefore increases complications such as postoperative bile reflux, alkaline reflux gastritis, and alkaline reflux esophagitis. When it comes to Braun anastomosis, it still fails to satisfy, because it provides limited improvement of complications. Meanwhile, Roux-en-Y can prevent alkaline reflux gastritis, but patients have an incidence of Roux-en-Y stasis syndrome (RSS), which consisted of upper abdominal distension, nausea, and vomiting, because the continuity of the small intestine is destroyed and the duodenal pacing impulse conduction is altered. ${ }^{4}$ RSS was reported in $\sim 30 \%$ of patients who undergo a Roux-en-Y syndrome. ${ }^{5}$

As an alternative method, the uncut Roux-en-Y (URY) has been developed, which performs both a Billroth II gastrojejunostomy plus a Braun anastomosis, and then blocks the jejunum input loop at the stump-jejunal anastomosis based upon the Billroth II and Braun anastomosis procedures. ${ }^{6}$ This prevents the bile and pancreatic juice from moving through the stomach-jejunum or anastomotic jejunum into the residual stomach. Also, the noncut jejunum could originate in the duodenum pacemaker impulse conduction in the meantime. Furthermore, this procedure could significantly reduce the alkaline reflux caused by cutting the vagus nerve and the absence of pylorus; this procedure even prevented the bile and pancreatic fluid from reaching the gastric or esophageal mucosa, reducing postoperative residual gastritis and reflux gastritis. ${ }^{7}$ Obviously the URY has advantages in reducing postoperative reflux gastritis theoretically. ${ }^{8}$

The specific operation of URY anastomosis is described as follows. First, a stomach-jejunum anastomosis is conducted from the Treitz ligament to the distal jejunum (25 $\mathrm{cm})$. Then the Braun anastomosis is performed in the input and output loop, which are $15 \mathrm{~cm}$ and $25-30 \mathrm{~cm}$ from the anastomosis of the jejunum, respectively. Finally, a nondiscrete closed jejunum at the input pouch from the gastrointestinal anastomosis $(5 \mathrm{~cm})$ is formed. Compared with other reconstructions, it is safe and feasible for URY to be performed laparoscopically because this surgery does not require interactions with the mesojejunm or any major vessels. Laparoscopic technologies have improved along with the optimization of the instruments, and it is a very convenient surgical method. Also, increasing understanding and use of this minimally invasive technology in specific procedures will hopefully make surgeons more willing to apply laparoscopic technology to radical gastrectomy. Nevertheless, there is no universal standard of digestive tract reconstruction after laparoscopic distal gastrectomy. In 1988, van Stiegman and Goff first presented the procedure of URY ${ }^{9}$ and reported that the URY reconstruction could divert bile and pancreatic juices through jejunojejunostomy. Uyama reported that laparoscopy-assisted URY reconstruction (nondiscrete closed jejunum at the input pouch from the gastrointestinal anastomosis $-10 \mathrm{~cm}$ ) could shorten the time of reconstruction compared with other procedures such as the Billroth II plus Braun anastomosis (BB), and there were zero events of alkaline reflux gastritis $(0 / 42) .{ }^{10}$ Ahn et al reported a study focusing on laparoscopic URY reconstruction $(n=22)$ and concluded that the average time of reconstruction was 25 minutes without postoperative complications. ${ }^{11}$ Current observational studies showed that both Roux-en-Y and URY had advantages over other methods in preventing reflux gastritis. ${ }^{12}$ And compared with the traditional Roux-en-Y, URY could reduce the morbidity rate of RSS, but this difference was not statistically significant. Additionally, URY could also shorten the time of reconstruction and decrease bleeding during the surgery. Above all, the optimal evidence showed that URY is a procedure of the Billroth II gastrojejunostomy plus Braun anastomosis, blocking the jejunum input loop at the stump-jejunal anastomosis, preventing bile and pancreatic juice through the stomach-jejunum anastomotic jejunum into the residual stomach. Meanwhile, the noncut jejunum could still originate in the duodenum pacemaker impulse conduction. In addition, the procedure of URY is treated as a safe and reliable method, and it could play a role in the alternatives of digestive tract reconstruction.

\section{Methods and analysis}

This is a prospective, multicenter, two-arm randomized controlled trial (RCT) in which 210 patients will be randomly assigned by computer to two groups. The experimental group 
will receive laparoscopic URY. The control group will receive laparoscopic BB.

The trial will assess the efficacy and safety of URY. The rate of RSS and recanalization for patients undergoing totally laparoscopic URY was also calculated. Before starting the trial, the sample size will be calculated based upon previously published studies about laparoscopic radical gastrectomy, as well as the data and safety monitoring board. A complete checklist of items according to Standardized Protocol Items: Recommendations for Intervention Trials $(2013)^{13}$ is provided.

\section{Participant selection}

Patients diagnosed with gastric cancer and undergoing totally laparoscopic radical gastrectomy from the five hospitals listed in Table 1 will be recruited. In total, 210 eligible patients will be identified and randomized (1:1) to the URY group and BB group.

The inclusion criteria are as follows: 1 ) age range, $18-75$ years; 2) diagnosis of gastric cancer (cT 2-4a/uT 2-4a, N \pm , M0), based on the 14th edition of the Japanese Classification of Gastric Carcinoma; 3) provide informed consent; 4) no history of surgical operation of stomach; and 5) no others organ malignant tumors.

The exclusion criteria are as follows: 1) transfer to the open abdomen $(>10 \mathrm{~cm}) ; 2$ ) primary tumor invaded surrounding organs; 3 ) emergency surgery; 4) gastric surgery in 30 days (endoscopic submucosal dissection [ESD]/endoscopic mucosal resection [EMR]) and a history of gastric surgery and gastrointestinal anastomosis; 5) NRS2002 score $>5$, and/ or albumin $<30 \mathrm{~g} / \mathrm{L}$ ); 6) have other medical diseases, such as severe systemic inflammatory disease, diabetes, or chronic lung diseases; 7) participating in other ongoing clinical trials and/or still in periods of follow-ups of other clinical trials; 8) receiving preoperative neoadjuvant chemoradiotherapy; and 9) others reasons, such as serious mental illness, unable to communicate and unable to complete Patient Report Outcomes (PRO), etc.

\section{Treatment protocols}

This is a prospective, multicenter, two-arm RCT in which 210 patients will be randomly assigned to two groups. Patients in the experimental group will undergo laparoscopic URY, and patients in the control group will undergo laparoscopic BB. Participants in both groups will undergo similar perioperative interventions such as Enhanced Recovery After Surgery (ERAS) strategies. All study data will be recorded in an electronic database.

\section{Follow-up}

An outpatient follow-up plan will be carried out 3 and 6 months after the operation. To avoid influences from the clinicians, randomized generated process will be performed in the operation room by independent nurses who are not involved in the study before gastrointestinal anastomosis. We still consider that the participants cannot be blinded to the intervention as well as the clinicians responsible for patient care. Only data collection and analysis will be blinded. Figure 1 shows the trial flow chart.

\section{Perioperative managements}

Before surgery, gastroscopies, endoscopic biopsies, and computed tomography scans will be performed to confirm the tumor size and location, and patients with organic metastases will be excluded based on assessment by two experienced pathologists.

After surgery, the ERAS guidelines will be followed and all participants will receive preoperative education, preemptive and multimodal analgesia, early ambulation, and laparoscopic radical gastrectomy as part of the perioperative treatment. Briefly, during the postoperative period, fluid

Table I Five experienced surgical teams

\begin{tabular}{|l|l|l|l|}
\hline Number & Center & Institute and department & Role \\
\hline 01 & $\begin{array}{l}\text { Xijing Hospital, The Fourth Military Medical } \\
\text { University } \\
\text { Henan Cancer Hospital \& Zhengzhou } \\
\text { University Cancer Hospital, Zhengzhou } \\
\text { University } \\
\text { The Second Hospital of Lanzhou University, } \\
\text { Lanzhou University } \\
\text { The First Affiliated Hospital of Xinjiang } \\
\text { Medical University, Xinjiang Medical } \\
\text { University } \\
\text { Affiliated Hospital of Qinghai University, } \\
\text { Qinghai University }\end{array}$ & $\begin{array}{l}\text { Xijing Hospital of Digestive Disease } \\
\text { Surgery }\end{array}$ & $\begin{array}{l}\text { Department of Minimally Invasive } \\
\text { Surgery } \\
\text { Department of Gastrointestinal } \\
\text { Surgery }\end{array}$ \\
\hline
\end{tabular}




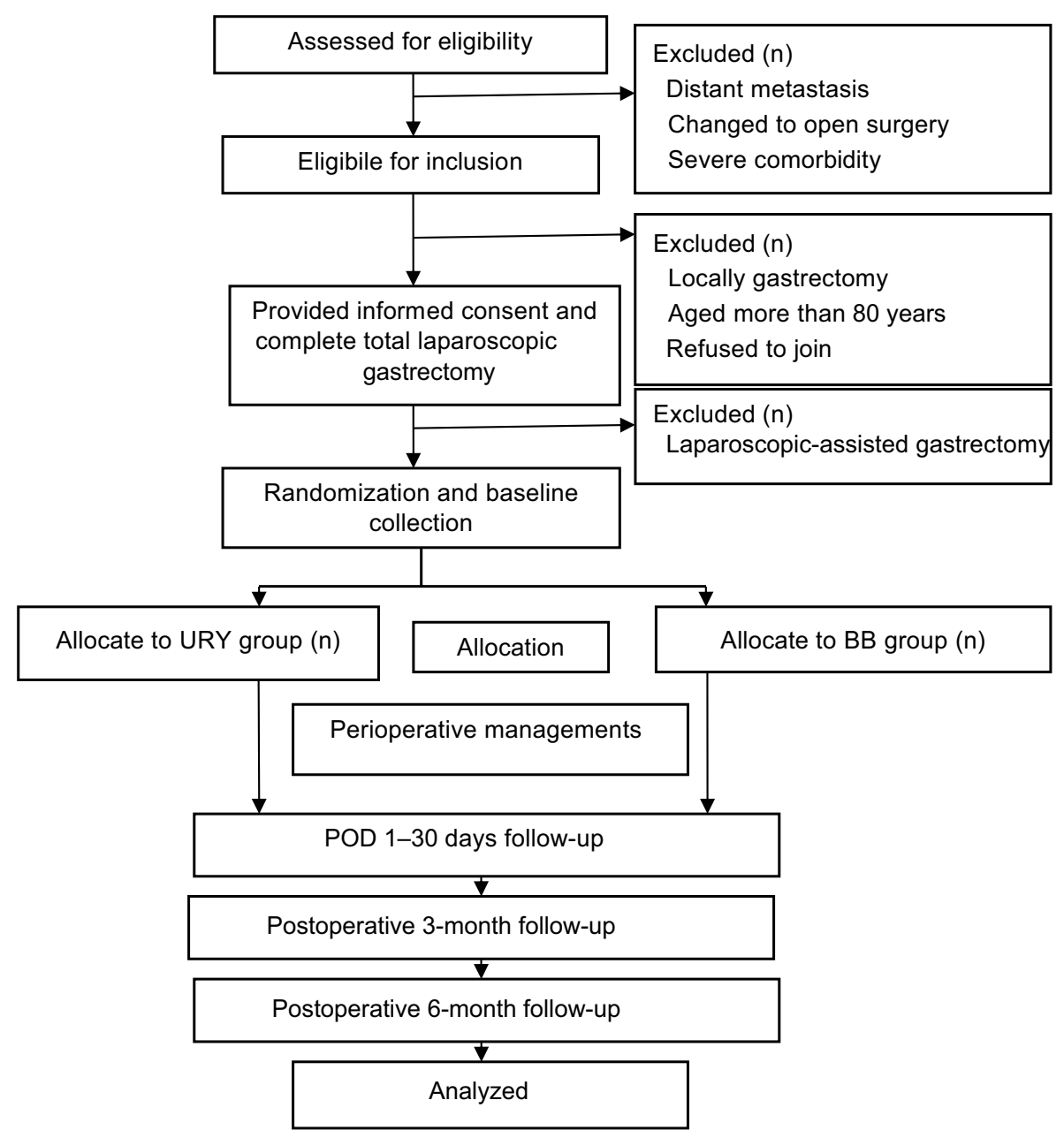

Figure I The trial flow chart.

Abbreviations: BB, Billroth II plus Braun anastomosis; POD, postoperative day; URY, uncut Roux-en-Y.

intake was from postoperative day (POD) 1 or 2, patients were advised to begin cautiously and increase intake according to tolerance. Furthermore, they were encouraged to take full semiliquid diet on POD 2 and normal food as soon as possible after surgery. If a patient is completely intolerant, the operators will decide whether early oral intake insist or not, and also the conventional way, including postoperative restricted oral intake, are considered to meet daily targets for mobilization. Furthermore, a nutritionist supervised the oral feeding during the trial using the diet principle of smaller and more frequent intake according to individual tolerance. Adequate caloric intake will be calculated using the HarrisBenedict formula (25-35 kcal $/ \mathrm{kg} /$ day). An investigator will be assigned to record the clinical observation data. Urinary bladder drainage was routinely used.

\section{Intervention protocols}

Laparoscopic radical gastrectomies will be performed by experienced surgical teams from the five centers listed in
Table 1, all of which have carried out this procedure with an annual caseload of $\sim 100$ gastric patients. The lymphadenectomy and main anastomosis will be completed laparoscopically, and the abdominal incision will be defined $<10 \mathrm{~cm}$. In terms of Billroth II procedure with Braun anastomosis in this protocol, first, a stomach-jejunum anastomosis is conducted from the Treitz ligament to the distal jejunum $(25 \mathrm{~cm})$, then the Braun anastomosis is performed in the input and output loop, which are generally 15 and $25 \mathrm{~cm}$ from the anastomosis of the jejunum, respectively. URY gastrojejunostomy is a modification of the Billroth II procedure with Braun anastomosis, which could be defined as an additional procedure of a nondiscrete closed jejunum at the input pouch from the gastrointestinal anastomosis $(5 \mathrm{~cm})$. All procedures will be completed laparoscopically except Braun anastomosis, which is decided by different surgeons following their own preference (linear stapler laparoscopically or manual anastomosis through small incision), and Figure 2 illustrates URY gastrojejunostomy after distal gastrectomy. 


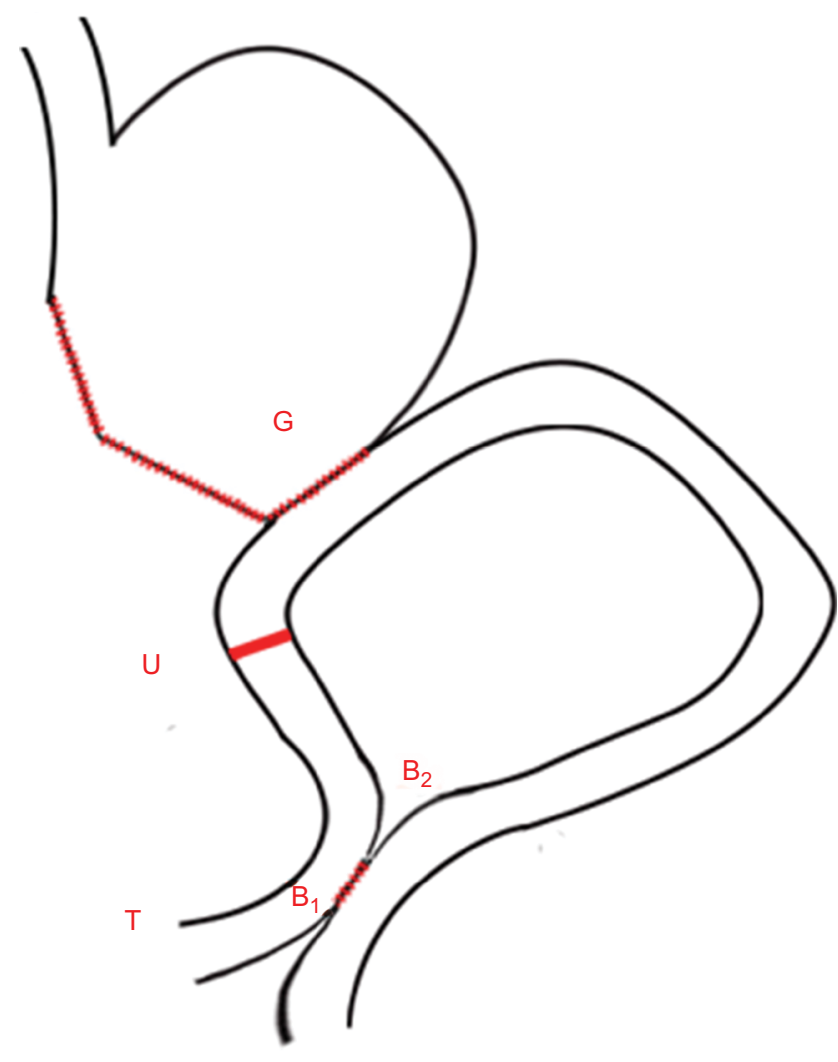

Figure 2 Uncut Roux-en-Y gastrojejunostomy after distal gastrectomy.

Notes: $G$ means gastrojejunostomy site; $B$ means Braun anastomosis site; $T$ means Treitz ligament. Distance between $T$ and $G$ is $25 \mathrm{~cm}$; distance between $T$ and $B_{1}$ is $10 \mathrm{~cm}$ and between $B_{1}$ and $B_{2}$ is more than $1.5 \mathrm{~cm}$; distance between $U$ and $G$ is 5 $\mathrm{cm}$; ditance between $G$ and $B\left(B_{1}\right.$ and $\left.B_{2}\right)$ is $25 \mathrm{~cm}$.

\section{Data collection/table schedule}

Once informed consent is signed, baseline data, such as age, gender, body mass index, and complications, will be collected by a clinical research assistant. The laboratory data $(\mathrm{pH}$ of gastric acid after POD1-3, total serum protein, serum albumin, and blood glucose) will also be tested preoperatively and during hospitalization to monitor the patients' nutritional status. A specified operator recorded the details of surgical procedures, such as operative approach, the location of tumor, lymph node metastasis, and pathological TNM stage.

From POD1-6, clinical observation data (anastomotic leakage, the time of first flatus and defecation, postoperative hospital stay, complications, etc) will also be recorded daily by an investigator to evaluate the postoperative recovery. The clinicians will be responsible for patient care and will not be involved in data collection. The criteria for discharge are as follows: tolerance of solid diet, return of bowel habits, and ability to walk on their own. Also, the contact information and address of each patient will be confirmed before their hospital discharge. Two follow-up visits will be carried out in the outpatient clinic or via telephone 3 and 6 months after discharge. These visits will focus on tolerance of the diet and any discomfort. The details of the time schedule are shown in Table 2.

\section{Statistical analysis}

The intention-to-treat principle will be applied in all analyses with an assumed drop-out rate. Normally distributed continuous variables will be described as the mean with corresponding SDs, and nonnormally distributed or categorical variables will be described as medians with the corresponding range or percentages and frequencies. Normally or nonnormally distributed continuous data will be compared by Student's $t$-test or the Mann-Whitney $U$-test, and the chi-squared test and Fisher's exact test will be used to compare categorical variables. With the exception of the primary outcome, secondary outcomes such as reflux gastritis, postoperative complications, time of first flatus and defecation, and postoperative length of stay will also be compared. Measurement data are expressed as mean \pm SD. The data will be analyzed using the independent Student's $t$-test, whereas categorical data will be calculated using the chi-squared test or Fisher's exact test. Data analysis will be performed using SPSS ${ }^{\circledR}$ software package version 22.0 (SPSS Inc., Chicago, IL, USA). $P<0.05$ is considered statistically significant.

\section{Study endpoints}

The primary outcome is the rate of alkaline reflux after laparoscopic radical gastrectomy, which will include two items: subjective items and objective items. One objective item is the $\mathrm{pH}$ of postoperative gastric acid $(>5 \mathrm{~mL})$, which will be tested randomly three times after POD1-3 and will also be assessed through gastroscopies and upper gastrointestinal radiography after 3 and 6 months. Subjective items are those included on the PRO ${ }^{14}$ Total scores of each symptom plus the frequency and the evaluated scales are shown in Table 3. And the guidelines of alkaline reflux or reflux gastritis assessed through gastroscopies are shown in Table 4.

The secondary outcomes are reconstruction time (minutes), blood loss (mL), RSS (\%), blocking recanalization (\%), first postoperative ventilation time (minutes), postoperative complications at 30 days per the Clavien Dindo classification, and the length of the postoperative hospital stay (days).

\section{Sample size estimate}

Previous studies reported the rate of reflux gastritis is $43.3 \%-67.7 \%,{ }^{15}$ and basing upon this rate, we make the hypothesis of $45 \%$ in the control group (BB procedure $45 \%)$. The parameters were designed to a noninferiority test 
Table 2 The details of the time schedule

\begin{tabular}{|c|c|c|c|c|c|c|}
\hline Outcomes & PODO & PODI & POD2 & POD3 & 3 months & 6 months \\
\hline Reflux gastritis (\%)* & \multicolumn{4}{|c|}{$\begin{array}{l}\text { Randomly select gastric acid }(>5 \mathrm{~mL}) \text { three times and test their } \mathrm{pH} \\
\text { after PODI-3 \& Patient Report Outcomes (PRO) }\end{array}$} & \multicolumn{2}{|c|}{$\begin{array}{l}\text { Gastroscopies/ } \\
\text { upper gastrointestinal } \\
\text { radiography }\end{array}$} \\
\hline $\begin{array}{l}\text { Reconstruction } \\
\text { time (minutes) }\end{array}$ & $※$ & - & - & - & - & - \\
\hline Blood Loss (mL) & $※$ & - & $\ldots$ & - & _ & - \\
\hline RSS (\%) & $※$ PRO & $※$ PRO & $※$ PRO & $※$ PRO & \multicolumn{2}{|c|}{$\begin{array}{l}\text { Gastroscopies/ } \\
\text { upper gastrointestinal } \\
\text { radiography }\end{array}$} \\
\hline $\begin{array}{l}\text { Blocking } \\
\text { recanalization (\%) }\end{array}$ & - & - & - & - & \multicolumn{2}{|c|}{$\begin{array}{l}\text { Gastroscopies/ } \\
\text { upper gastrointestinal } \\
\text { radiography }\end{array}$} \\
\hline $\begin{array}{l}\text { First postoperative } \\
\text { ventilation time } \\
\text { (minutes) }\end{array}$ & $※$ & $※$ & $※$ & $※$ & - & - \\
\hline $\begin{array}{l}\text { Postoperative } \\
\text { complications (n) }\end{array}$ & $※$ & $※$ & $※$ & $※$ & \multicolumn{2}{|c|}{$\begin{array}{l}\text { Till postoperative I } \\
\text { month }\end{array}$} \\
\hline $\begin{array}{l}\text { Postoperative } \\
\text { hospital stay (d) }\end{array}$ & $※$ & $※$ & $※$ & $※$ & \multicolumn{2}{|c|}{$\begin{array}{l}\text { Till postoperative } \\
\text { hospital stay }\end{array}$} \\
\hline
\end{tabular}

Notes: *Primary outcomes. ※Needing to conduct at this time point. _No need to conduct at this time point.

Abbreviations: POD, postoperative day; RSS, Roux-en-Y stasis syndrome.

Table 3 The evaluated scales and frequency of Patients Report Outcomes (PRO)

\begin{tabular}{|c|c|c|c|c|c|}
\hline & Epigastric pain & Heartburn & Biliary vomiting & Postprandial bloating & Nausea \\
\hline 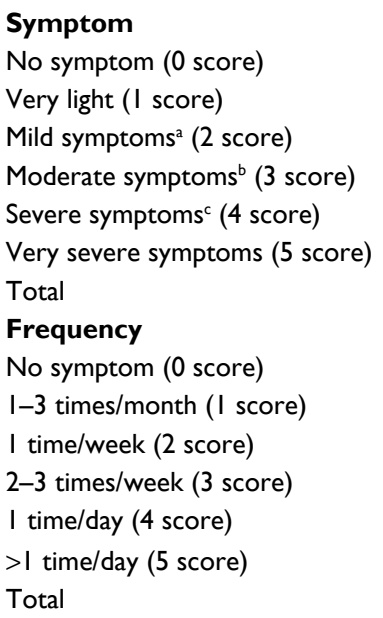 & & & & & \\
\hline
\end{tabular}

Notes: aNoticed only when the doctor reminds. ${ }^{b}$ Do not affect daily life. ${ }^{c}$ Can affect normal life.

Table 4 The guidelines of reflux gastritis assessed through gastroscopies

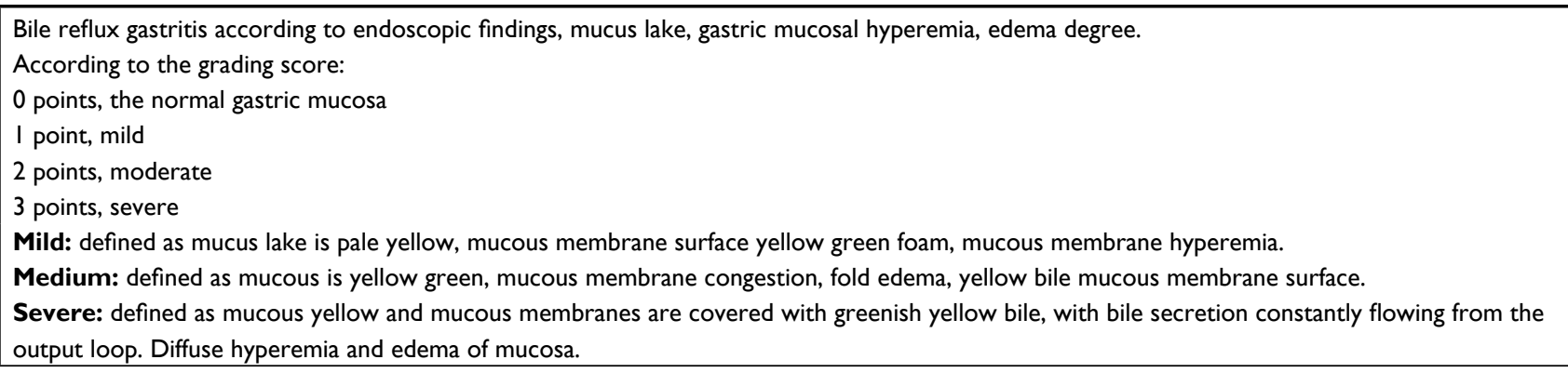


with a noninferiority margin of $20 \%(\alpha=0.05, \beta=0.20$, and a power of $80 \%$ ). Considering both clinical and statistical considerations, no less than 172 participants (86 participants in each group) will be needed. Allowing for a 20\% drop-out, a total of 210 participants (105 participants in each group) will be recruited.

\section{Strengths and limitations of this study}

The feasibility of URY has not been established in prospective randomized studies. This trial will be the first RCT to assess URY for patients with advanced gastric cancers suffering from totally laparoscopic radical distal gastrectomy.

The primary outcome is the rate of reflux gastritis after totally laparoscopic radical gastrectomy, which will include two items: subjective items and objective items. One objective item is the $\mathrm{pH}$ of postoperative gastric acid $(>5 \mathrm{~mL})$, which will be tested randomly three times after POD 1-3, and specifically, PRO will be recorded of subjective items.

One limitation is that a minority of patients may have no gastric fluid in their nasogastric tube and therefore could not be tested for postoperative gastric acid.

Another limitation is that this study only assessed the feasibility of URY and BB in patients undergoing laparoscopic radical distal gastrectomy; it did not compare uncut URY with traditional Roux-en-Y, which should be assessed in further studies.

\section{Ethics and dissemination}

Ethics approval has been sought from the Ethics Committee at the First Affiliated Hospital (Xijing Hospital) of Fourth Military Medical University (KY20172001-1). Before allowing participation in the study, written informed consent is obtained from each participant and their guardians, primary caregivers, and research assistants. The results of this study will be disseminated at several research conferences and as published articles in peer-reviewed journals. The present study protocol was prepared in accordance of the Standardized Protocol Items: Recommendations for Intervention Trials statement. This trial has been registered at Chinese Clinical Trial Registry: ChiCTR-INR-17010594 (registration date: 9 February, 2017).

Ethical clearance has been obtained from Ethics Committee at the First Affiliated Hospital (Xijing Hospital) of Fourth Military Medical University (KY20172001-1) and was obtained similarly at the others centers as needed. Written informed consent was obtained from the patient before screening. The results of this study will be disseminated at several research conferences and as published articles in peer-reviewed journals.

\section{Trial status}

The enrollment of this study is ongoing at the time of manuscript submission.

\section{Acknowledgment}

This work was supported by Wu Jie-Ping Medical Foundation (320.6750.17508). We thank the assistance of a professional copyediting agency, MogoEdit, to improve the quality of language in our manuscript.

\section{Author contributions}

GJ, YF, CW, WBZ, SY, and SQJ, as the main surgeon, performed each operation in each center. QW, KLY, QRN, JY, DZ, QM, and QYW were the main investigators and data recorders. KLY, QRN, and JY tested the feasibility of the study. QW KLY and GJ wrote the manuscript. All authors approved the final version of the manuscript. All authors contributed to the research protocol design, drafting and revising the protocol, gave final approval of the version to be published, and agree to be accountable for all aspects of the work.

\section{Disclosure}

The authors report no conflicts of interest in this work.

\section{References}

1. Torre LA, Bray F, Siegel RL, Ferlay J, Lortet-Tieulent J, Jemal A. Global cancer statistics, 2012. CA Cancer J Clin. 2015;65(2):87-108.

2. Ji JF, Hu X, Chen L, Sun YH. [Expert consensus on digestive tract reconstruction after gastrectomy]. Chin J Pract Surg. 2014;34:217-221. Chinese.

3. Hoya Y, Mitsumori N, Yanaga K. The advantages and disadvantages of a Roux-en-Y reconstruction after a distal gastrectomy for gastric cancer. Surg Today. 2009;39(8):647-651.

4. Gustavsson S, Ilstrup DM, Morrison P, Kelly KA. Roux-Y stasis syndrome after gastrectomy. Am J Surg. 1988;155(3):490-494.

5. Yang L, Zk X, Xu H, Zhang DC. [Total laparoscopic uncut Roux-en-Y anastomosis in the distant gastrectomy with D1+ or D2dissection for gastric cancer]. Chin J Pract Surg. 2015;201535(35):10991099-11021102. Chinese.

6. Xue K, Zy L, Shan F, et al. [V-Loc based uncut Roux-en-Y reconstruction in totally laparoscopic distal gastrectomy: a report of 7 patients]. Chin J Pract Surg. 2015;35:1095-1098. Chinese.

7. Zou ZY, Xh D, Li R. [Progress of application of Uncut Roux-en-Y gastrojejunostomy after distal gastrectomy for gastric cancer]. J Clin Surg. 2016;24:237-239. Chinese.

8. Zhang YM, Liu XL, Xue DB, Wei YW, Yun XG. Myoelectric activity and motility of the Roux limb after cut or uncut Roux-en-Y gastrojejunostomy. World J Gastroenterol. 2006;12(47):7699-7704.

9. Huang Y, Wang S, Shi Y, et al. Uncut Roux-en-Y reconstruction after distal gastrectomy for gastric cancer. Expert Rev Gastroenterol Hepatol. 2016;10(12):1341-1347.

10. Uyama I, Sakurai Y, Komori Y, et al. Laparoscopy-assisted uncut Rouxen-Y operation after distal gastrectomy for gastric cancer. Gastric Cancer. 2005;8(4):253-257.

11. Ahn SH, Son SY, Lee CM, Jung DH, Park Doj, Kim HH. Intracorporeal uncut Roux-en-Y gastrojejunostomy reconstruction in pure singleincision laparoscopic distal gastrectomy for early gastric cancer: unaided stapling closure. J Am Coll Surg. 2014;218(1):e17-e21. 
12. Park JY, Kim YJ. Uncut Roux-en-Y Reconstruction after laparoscopic distal gastrectomy can be a favorable method in terms of gastritis, bile reflux, and gastric residue. J Gastric Cancer. 2014;14(4): 229-237.

13. Chan AW, Tetzlaff JM, Altman DG, et al. SPIRIT 2013 statement: defining standard protocol items for clinical trials. Ann Intern Med. 2013;158(3):200.
14. Cleeland CS, Sloan JA, Group AO, ASCPRO Organizing Group. Assessing the Symptoms of Cancer Using Patient-Reported Outcomes (ASCPRO): searching for standards. J Pain Symptom Manage. 2010;39(6):1077-1085.

15. In Choi C, Baek DH, Lee $\mathrm{SH}$, et al. Comparison between Billroth-II with braun and roux-en-Y reconstruction after laparoscopic distal gastrectomy. J Gastrointest Surg. 2016;20(6):1083-1090.

\section{Publish your work in this journal}

Cancer Management and Research is an international, peer-reviewed open access journal focusing on cancer research and the optimal use of preventative and integrated treatment interventions to achieve improved outcomes, enhanced survival and quality of life for the cancer patient. The manuscript management system is completely online and includes a very quick and fair peer-review system, which is all easy to use. Visit http://www.dovepress.com/testimonials.php to read real quotes from published authors. 